\title{
Calcium With and Without Magnesium for Primary Dysmenorrhea: A Double-Blind Randomized Placebo- Controlled Trial
}

\author{
Sakineh Mohammad-Alizadeh Charandabi ${ }^{1}$, Mojgan Mirghafourvand ${ }^{1}$, Salimeh Nezamivand-Chegini ${ }^{2 *}$, \\ Yousef Javadzadeh ${ }^{3}$
}

\begin{abstract}
Objectives: To assess the effect of co-administration of calcium and magnesium and calcium alone on pain intensity in primary dysmenorrhea.

Materials and Methods: In this study, 63 students with primary dysmenorrhea were randomly allocated into 2 intervention (receiving one tablet a day combined $600 \mathrm{mg}$ calcium carbonate and $300 \mathrm{mg}$ magnesium stearate, or only $600 \mathrm{mg}$ calcium carbonate from 15th day of cycle until no pain day on the following cycle) and 1 placebo groups. The subjects filled in a diary including pain intensity ( 0 - 10 visual analog scale) and rest length for 2 cycles before intervention and 2 cycles under intervention. Univariate general linear model was used for data analysis.

Results: One from each intervention groups were lost to follow up. Both combined calcium- magnesium and calcium alone groups had better outcomes than the placebo group in pain intensity (adjusted difference $-1.9,95 \%$ CI -2.2 to -1.7 and $-0.9,-1.2$ to -0.6 , respectively), rest length ( -2.5 hours, -3.2 to -1.9 and $-1.7,-2.4$ to -1.1$)$ and number of Ibuprofen taken $(-2.6,-4.1$ to -1.1 and $-1.5,-2.9$ to -0.1). The calcium-magnesium group had significantly better outcomes than the calcium group in pain relief and rest length but not in the Ibuprofen group. No side effect was reported.

Conclusion: Both combined calcium-magnesium and calcium alone are effective in relieving the pain and decreasing the rest length due to primary dysmenorrhea, but better efficacy was obtained from combined calcium and magnesium in comparison with its counterpart.

Keywords: Calcium, Dysmenorrhea, Magnesium, Pain
\end{abstract}

\section{Introduction}

Primary dysmenorrhea is menstrual pain which occurs in the absence of demonstrable pelvic disease and occurs in about $50 \%$ of adult women and $90 \%$ of teens (1). It affects women's quality of life and in severe cases can cause weakness and inefficiency (2).

Although the exact cause of primary dysmenorrhea is unknown, but the accepted theory about its cause is over-production of uterine prostaglandins (3). Prostaglandin inhibitors are effective in its treatment in about $80 \%$ of cases but their long-term use may cause some side effects like nausea, stomach irritation, ulcers, renal papillary necrosis and decrease in renal blood flow $(4,5)$. Thus nowadays, most consumers are looking for alternative pain relievers with minimal or no adverse effects (6).

There is some evidence supporting the role of some dietary supplements, including Omega-3 fatty acids, such as fish oil, vitamin $\mathrm{B} 1$, vitamin $\mathrm{B} 6$, vitamin $\mathrm{D}$, vitamin $\mathrm{E}$, magnesium and vitamin $\mathrm{B} 6$, on menstrual pain relieve. Calcium may also help reduce menstrual pain but there is no clear evidence in this regard (6).

Benefits of calcium supplements on reducing the severity of menstrual symptoms including abdominal cramps, general aches and back pain have been shown in some studies (7-9). Disruption of calcium regulation has been proposed as an underling factor for increasing incidence and severity of menstrual pain $(10,11)$. It may affect them through controlling neuromuscular activities (12).

Also, there are some possible pathways by which magnesium might influence dysmenorrhea. It relaxes the muscles and is involved in the activity of serotonin and other neurotransmitters, as well as in vascular contraction, neuromuscular function and cell membrane stability (13). Some studies show that magnesium has positive effect on reducing premenstrual syndrome symptoms (14-16), and severity of primary dysmenorrhea $(17,18)$. The researchers reported evidence of reduced levels of prostaglandin F2 $\alpha$, a hormone-like substance involved in pain and inflammation (19). Also, it may affect them by decreasing prostaglandins (20-22), by activating B vitamins, especially vitamin $\mathrm{B} 6$ and/or affecting muscle relaxation by controlling the effect of calcium on muscle contraction (23).

To the best of our knowledge, this is the first study aimed to determine the effect of combined administration of calcium and magnesium and calcium alone on the pain intensity and rest length due to dysmenorrhea (primary 
outcome) and number of analgesics taken (secondary outcome) in students residing in dormitories, Tabriz, Iran.

\section{Materials and Methods}

The study was a double blind, placebo-controlled and parallel-group study with balanced randomization [1:1:1] for the 3 groups ( 2 intervention groups and 1 control group), and conducted on students residing in 3 dormitories (2 dormitories of Tabriz University of Medical Sciences and 1 dormitory of the University of Tabriz) located in Tabriz, Iran from February 2012 to October 2012.

The specific research questions were as follows: (a) Does co-administration of calcium and magnesium and calcium alone decrease the intensity of pain and rest length (primary outcome) in primary dysmenorrhea? (b) Does co-administration of calcium and magnesium and calcium alone decrease the use of analgesics (secondary outcome) in primary dysmenorrhea? (c) Which intervention is more effective in reducing pain with regard to primary and secondary outcomes of study? And $(d)$ what are the side effects of each intervention?

In the current study, we tested the hypothesis that both intervention groups can ameliorate the intensity of pain and reduce the rest length and use of analgesics due to dysmenorrhea.

Female college students experiencing primary dysmenorrhea in the above mentioned settings were included in the study with the following criteria: $(a)$ having regular menstrual periods, $(b)$ having moderate or severe primary dysmenorrhea (pain score of 5 to 9 on visual analog scale in the previous cycles), (c) free from medical and gynecological problems and $(d)$ being single. Students with history of any chronic diseases, or those using oral contraceptive pills or vitamin supplements were excluded. Considering 6.8 for mean and 1.4 for standard deviation (SD) of menstrual pain intensity based on results of a study carried out at the same setting (6), $\alpha=0.05, \beta=0.10$ and $15 \%$ possible drop out, sample size was calculated 20 for each group to detect at least $25 \%$ reduction in mean of pain intensity due to the intervention.

Data were collected using an interview questionnaire that was designed by the researchers to collect the necessary data. The questionnaire was divided into 2 parts as follows: (a) Socio-demographic data, (2) Menstrual and reproductive characteristics data. The second tool is the standard questionnaire of pain (VAS, visual analogue scale) for assessment of menstrual pain. It is a validated scale length of $10 \mathrm{~cm}$ with 0 (no pain) on the left end and 10 (the maximum imaginable pain) on the right end (24). Data collection took place within 4 months from February 2012 to October 2012. Permission was obtained from the director of dormitories.

Each combined tablet contained $300 \mathrm{mg}$ magnesium stearate, $600 \mathrm{mg}$ calcium carbonate and also some lactose, and micro-crystalline cellulose as excipient. Each calcium tablet contained $600 \mathrm{mg}$ calcium carbonate, lactose and micro-crystalline cellulose. Placebo had the same ingredients except calcium and magnesium. The tablets were identical in color, shape and size and were produced in industrial pharmacy laboratories of Faculty of Pharmacy, Tabriz University of Medical Sciences under direct supervision of the pharmacist from our research team.

In this study, in order to select the subjects, the researcher attended the students' rooms in the selected dormitories. The dormitories were selected randomly but subjects at the dormitories were recruited by convenience sampling. The aims of study were explained to the students in each room and those willing participate gave positive answer to the question "Are you suffering from painful menstruation?" to fill in a questionnaire containing questions designed to determine the eligible persons.

All the students with primary dysmenorrhea were invited to participate in the preliminary recruitment. A total of 80 eligible students were enrolled into the study after signing a written informed consent. We did not recruit more than one person from each dormitory room due to possible pooling of the pills given to the subjects. We gave the participants 20 ibuprofen tablets $400 \mathrm{mg}$ and a diary to fill in following 2 consecutive menstrual cycles. We asked them to take the pills only for menstrual pain relief, if needed, and not to take any other pain reliever for the pain and record every taken pill in the diary. The diary also included a table to record pain intensity and rest length (minutes) due to the pain during 2 days before and 3 days after start of menstruation. Out of the 80 students, 63 who had good co-operation, i.e. had completed the pre-intervention diaries fully and correctly and were willing to continue participation in the study, were randomly allocated into 3 groups with allocation ratio of 1:1:1 to receive combined calcium-magnesium, only calcium or placebo tablets (Figure 1).

We gave each participant the package based on the sequence determined at the enrolling visit. Also $20 \mathrm{ibu}-$ profen tablets ( $400 \mathrm{mg}$ ) and a second diary were given to them. The diary included the same pre-intervention items in addition to 2 extra items about record of taking the intervention pills and report any unwanted adverse effects including diarrhea, upset stomach and heartburn in menstrual cycles including duration of menses and interval of cycles. We asked the participants to take the intervention pills regularly, 1 pill a day, from the day 15 th of their cycle till the day with no menstrual pain in the following cycle and record the pill taken daily in the diary. We also instructed them to take the ibuprofen pills when they need and fill in the other parts of the diary exactly like the pre-intervention period for 2 consecutive under-intervention cycles. The completed diary with the remaining pills (both the pills given as intervention and the ibuprofen) were gathered after 2 months.

A person not involved in the participant recruitment, data collection and analysis, determined the allocation sequence using a computerized randomization program considering block randomization with block sizes of 6 and 9. The person also prepared sequentially the numbered identical packages containing the 40 intervention pills (for using during 2-cycles) based on the allocation sequence. 


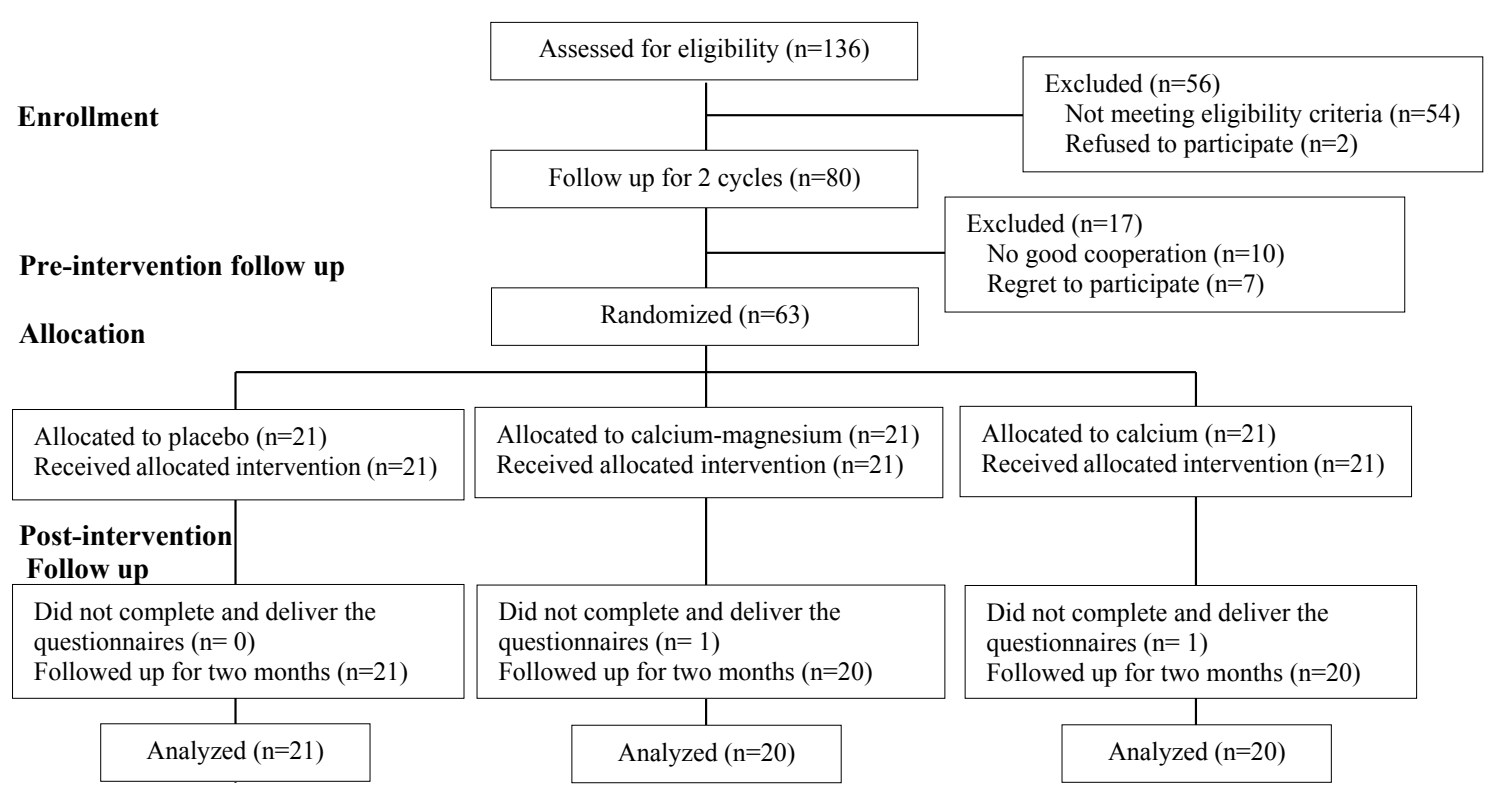

Figure 1. Flowchart of Patients Through the Trial.

Primary outcomes of this study were pain intensity score and rest length due to dysmenorrhea and secondary outcome was number of analgesics (ibuprofen) taken during 2 menstrual cycles. Also, volume of menstrual bleeding and intensity of premenstrual symptoms were recorded as secondary outcomes.

Menstrual pain intensity score at each cycle was calculated in 2 ways: (a) Mean pain intensity during 2 days, the days with higher pain intensity out of the 5 days ( 2 days before and 3 days after start of menstruation); (b) Mean pain intensity during the 5 days. The rest length at each cycle was calculated by sum of the recorded rest due to pain during the 5 days.

Average of the outcomes during the 2 pre-intervention cycles was considered as baseline values and average of those recorded during the 2 cycles after starting the intervention were considered as under-intervention values.

\section{Statistical Analysis}

Normality of distribution of the pain intensity score, rest length and number of the analgesic pills taken by study group was checked using the Kolmogorov-Smirnov test which all except number of ibuprofen taken, were normal. As different number of ibuprofens taken during under-intervention and baseline period was distributed normally, we used the difference for comparing the groups to identify the effect of intervention on this outcome.

One-way analysis of variance (ANOVA) was used for comparison of the baseline values between the groups. After controlling for assumption of the model, univariate general linear model was used for comparison of the groups in terms of the under-intervention pain intensity and rest length was adjusted for the baseline values. Sidak was used for multiple comparisons between the groups. All analyses were done using SPSS version 13.0 and $P<0.05$ was considered as statistically significant difference.

\section{Results}

Of the 63 students randomized into the groups, 2 (one from each treatment group) did not complete the diaries and were excluded from analyses. No side effect was reported by any of the subjects in the groups. There was no considerable difference between the groups in any of the baseline characteristics. Mean \pm SD of their age was $21.0 \pm 2.2$ and their body mass index (BMI) was $22.3 \pm 3.0$. Two-thirds aged 18-21 years and about three-fourths (79\%) had normal BMI (20.0-25.0). About one-fourth (26\%) mentioned regular exercise. About two-thirds (69\%) reported a family history of dysmenorrhea and more than half (54\%) reported that their menstrual pain intervened often or always with their usual activities (Table 1).

At the baseline, mean \pm SD of the pain intensity score during the 2 days with highest score was $5.4 \pm 2.2$; and during the 5 days was $2.7 \pm 1.6$; mean \pm SD of the rest length was $4.2 \pm 2.5$ hours; and the number of ibuprofen taken was $3.3 \pm 2.2$. There were no significant differences between the groups in any of them.

Participants in both combined calcium and magnesium, and only calcium groups had better outcomes than the placebo group with regard to both the primary outcomes; i.e., the 2-day pain intensity (adjusted difference [95\% CI]: -1.9 [-2.2 to -1.7$]$, and -0.9 [-1.2 to -0.6$]$, respectively) and rest length (-2.5 [-3.2 to -1.9$]$ and -1.7 [-2.4 to -1.1$]$, respectively), as well as in the secondary outcome, i.e., difference number of ibuprofen taken [mean difference [ $95 \%$ $\mathrm{CI}$ ], $-2.6[-4.1$ to -1.1$]$ and $-1.5[-2.9$ to -0.1$]$, respectively). Participants in combined calcium and magnesium group had better outcomes than the only calcium group in pain relief $(-1.1[-1.5$ to -0.7$])$ and resting time $(-0.8[-1.5$ to $-0.1])$ and no significant difference was detected in regard 
Table 1. Baseline Characteristics of the Subjects in the Study Groups

\begin{tabular}{|c|c|c|c|}
\hline Characteristics & Calcium-Magnesium ( $n=20)$ & Calcium $(n=20)$ & Placebo $(n=21)$ \\
\hline Age in years, mean \pm SD & $21.0 \pm 2.0$ & $21.3 \pm 2.5$ & $20.8 \pm 2.2$ \\
\hline $\mathrm{BMI}$ in $\mathrm{kg} / \mathrm{m}^{2}$, mean $\pm \mathrm{SD}$ & $22.3 \pm 2.8$ & $23.5 \pm 3.6$ & $21.3 \pm 2.1$ \\
\hline \multicolumn{4}{|l|}{ Educational level, No. (\%) } \\
\hline Bachelor of science & $7(35)$ & $10(50)$ & $8(38)$ \\
\hline Higher & $13(65)$ & $10(50)$ & $13(62)$ \\
\hline Regular exercise & $4(20)$ & $7(35)$ & $5(24)$ \\
\hline Age at menarche in years, mean \pm SD & $12.9 \pm 1.3$ & $12.9 \pm 0.9$ & $13.1 \pm 1.5$ \\
\hline Family history of dysmenorrhea, No. (\%) & $10(71)$ & $14(70)$ & $12(67)$ \\
\hline Interference with daily activities, often/always, No. (\%) & $13(65)$ & $8(40)$ & $12(57)$ \\
\hline
\end{tabular}

to the difference in the number of ibuprofen taken $(-1.1$ [-2.6 to 0.3$]$ ) (Table 2).

We also compared the groups in terms of mean of the pain intensity score during the 5 days; the results were similar with the 2-day pain intensity score, i.e. the adjusted difference $(95 \% \mathrm{CI})$ was $-1.1(-1.4$ to -0.8$)$ and $-0.5(-0.8$ to -0.2) comparing the combined calcium and magnesium, and only calcium groups with the placebo group and it was $-0.5(-0.8$ to 0.2$)$ comparing the 2 treatment groups with each other.

We also used repeated measures general linear model to see whether or not there is any difference between effect size of the treatments in the first and the second under-intervention cycles in terms of the outcomes. The results showed no statistically significant difference between the 2 periods in terms of any of the outcomes (no interaction between period and the effect).

\section{Discussion}

This study was conducted to investigate the effect of administration of calcium alone and combined with magnesium on primary dysmenorrhea. The results showed that taking calcium alone or co-administration with magnesium reduce the severity of menstrual pain and rest length due to the pain and number of analgesics taken. Moreover, this supplementation seems to be well tolerated and safe, with no reported adverse event. The obtained efficacy was higher with combination of calcium and magnesium compared with calcium alone.

Dysmenorrhea is the most common problem in women with normal ovulatory cycles and no pelvic pathology which occurs in $20 \%-90 \%$ in adolescents and young females (25). So, finding appropriate and effective alternatives for its treatment is important. The most common treatment for relieving primary dysmenorrhea is use of nonsteroidal anti-inflammatory drugs (NSAIDs) with failure rate of about $20 \%-25 \%$, as well as occurrence of certain side effects (26). Therefore, many researchers are trying to find other treatments such as complementary and alternative therapies and numerous studies are being conducted on this matter.

Nutrition is one of the most important factors influenc- ing the quality of life. Nutritional and metabolic conditions may have an important role in the etiology and treatment of menstrual disorders. Menstrual pain is relieved by good diet (27). The regulation of nutrient intake often changes across the menstrual cycle and is affected by the menstrual cycle hormone, dietary treatments are numerous and still require more investigations (28).

According to our bibliography studies, this is the first study aimed to examine the effect of calcium supplementation alone on pain intensity and rest length in primary dysmenorrhea. Therefore, there is no other study for comparing the results directly. However, some studies suggest indirectly that calcium can be effective in reducing the severity of dysmenorrhea. It has been reported that plasma levels of calcium in premenstrual period is lower in people suffering from premenstrual syndrome $(29,30)$. Di Cintio et al reported a relationship between calcium intake in food and reducing the severity of primary dysmenorrhea (31). In another study done by Thys-Jacobs et al, taking $1200 \mathrm{mg} / \mathrm{d}$ calcium carbonate for 3 cycles reduced menstrual symptoms including lower back pain and abdominal cramping and the reduction was significantly higher than the placebo group (9).

Also, a small randomized trial showed that increasing dietary calcium intake reduce mood and pain symptoms associated with menstruation (7). Mechanism of action of calcium in reducing pain is not clearly known. Calcium deficiency may cause increased pain and pre-menstrual symptoms. Therefore it can be concluded that calcium intake may reduce the severity of menstrual pain and associated symptoms.

No study was found on the effect of combined calcium and magnesium on pain intensity and rest length in primary dysmenorrhea. Positive effect of magnesium supplementation on pain reduction of women with primary dysmenorrhea has been shown in some clinical trials (14, 17,18). Also, Hudson showed that increasing intake of dietary magnesium in girls with primary dysmenorrhea can reduce the severity of dysmenorrhea (32). However, a study showed no positive effect of taking daily 500 $\mathrm{mg}$ magnesium for 5 consecutive cycles on the pain relief compared with placebo. In this study vitamin B6 alone 
Table 2. Comparison of the Calcium-Magnesium, Only Calcium and Placebo Groups With Regard to the Primary and Secondary Outcomes

\begin{tabular}{|c|c|c|c|c|c|c|c|c|c|c|}
\hline & \multirow{2}{*}{$\begin{array}{c}\begin{array}{c}\text { Calcium \& } \\
\text { Magnesium }(n=20)\end{array} \\
\text { Mean } \pm S D\end{array}$} & \multirow{2}{*}{ 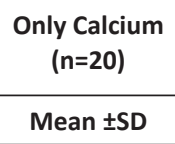 } & \multirow{2}{*}{$\begin{array}{c}\begin{array}{c}\text { Placebo } \\
(n=21)\end{array} \\
\text { Mean } \pm S D\end{array}$} & \multirow{2}{*}{$\begin{array}{c}\text { Comparison of } \\
3 \text { Groups }\end{array}$} & \multicolumn{2}{|c|}{$\begin{array}{l}\text { Comparison of Calcium \& } \\
\text { Magnesium With Placebo }\end{array}$} & \multicolumn{2}{|c|}{$\begin{array}{c}\text { Comparison of Only Calcium } \\
\text { With Placebo }\end{array}$} & \multicolumn{2}{|c|}{$\begin{array}{l}\text { Comparison of Calcium \& } \\
\text { Magnesium With Only Calcium }\end{array}$} \\
\hline & & & & & Difference $(95 \% \mathrm{Cl})$ & $P$ Value & Difference $(95 \% \mathrm{Cl})$ & $P$ Value & Difference $(95 \% \mathrm{Cl})$ & $P$ Value \\
\hline \multicolumn{11}{|c|}{ Primary Outcome } \\
\hline \multicolumn{11}{|c|}{ Pain intensity (VAS, 0-10) a } \\
\hline Baseline $^{b}$ & $6.0 \pm 2.3$ & $5.2 \pm 2.0$ & $5.4 \pm 2.2$ & 0.45 & & & & & & \\
\hline Under-intervention $^{c}$ & $3.9 \pm 2.1$ & $4.2 \pm 2.0$ & $5.3 \pm 2.2$ & $<0.001$ & $-1.9(-2.2$ to -1.7$)$ & $<0.001$ & $-0.9(-1.2$ to -0.6$)$ & $<0.001$ & $-1.1(-1.4$ to -0.8$)$ & $<0.001$ \\
\hline \multicolumn{11}{|c|}{ Rest length due to dysmenorrhea at each cycle (h) } \\
\hline Baseline & $4.8 \pm 2.8$ & $4.0 \pm 2.7$ & $4.0 \pm 2.2$ & 0.552 & & & & & & \\
\hline Under-intervention & $1.2 \pm 1.3$ & $1.5 \pm 1.9$ & $3.2 \pm 2.2$ & $<0.001$ & $-2.5(-3.2$ to -1.9$)$ & $<0.001$ & $-1.7(-2.4$ to -1.1$)$ & $<0.001$ & $-0.8(-1.5$ to -0.1$)$ & 0.016 \\
\hline \multicolumn{11}{|c|}{ Secondary Outcome } \\
\hline
\end{tabular}

Number of Ibuprofen taken at each cycle ${ }^{d}$

\begin{tabular}{|c|c|c|c|c|c|c|c|c|c|c|}
\hline Baseline $^{b}$ & $3.8 \pm 2.3$ & $3.0 \pm 1.9$ & $3.2 \pm 2.4$ & 0.754 & & & & & & \\
\hline Under-intervention & $0.6 \pm 1.1$ & $1.2 \pm 2.3$ & $4.6 \pm 3.9$ & & & & & & & \\
\hline Difference ${ }^{e}$ & $-3.5 \pm 2.0$ & $-2.4 \pm 1.1$ & $-0.9 \pm 2.2$ & $<0.001$ & $-2.6(-4.1$ to -1.1$)$ & 0.039 & $-1.5(-2.9$ to -0.1$)$ & 0.039 & $-1.1(-2.6$ to 0.3$)$ & 0.174 \\
\hline
\end{tabular}

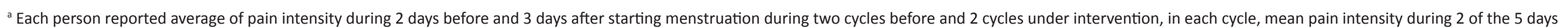
with highest pain intensity was considered as pain intensity at each cycle and mean of pain intensity during the 2 pre-intervention cycle considered as baseline pain intensity and mean intensity during two months after starting the intervention was considered as under-intervention pain intensity.

${ }^{\mathrm{b}}$ One-way ANOVA was used for the baseline comparisons

' Univariate general linear model was used for the under-intervention pain intensity comparisons adjusted for the baseline values using Sidak for the multiple comparisons between the groups

${ }^{\mathrm{d}}$ Mean number of Ibuprofen $400 \mathrm{mg}$ taken at each cycle due to dysmenorrhea at the two pre-intervention cycle (baseline) and at 2 months after starting the intervention (under-intervention)

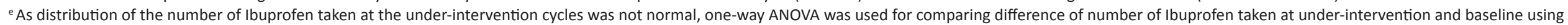
Sidak for the multiple comparisons between the groups 
was more effective than magnesium alone and also vitamin B6 combined with magnesium in reducing pain (23). In the present study, there was no group with taking magnesium alone. So, the results of this study cannot be judgmental about the effect of magnesium alone on primary dysmenorrhea.

A typical supplemental dosage of magnesium is $600 \mathrm{mg}$ daily. For premenstrual syndrome and dysmenorrhea, an alternative approach is to start taking 500 to $1000 \mathrm{mg}$ daily, beginning on day 15 of the menstrual cycle and continuing until menstruation begins (33). Daily need of calcium in girls in this age is $1000-1200 \mathrm{mg}$ and even a dose of $2500 \mathrm{mg} / \mathrm{d}$ was well tolerated and no side effects have been reported (34).

There are some reports indicating inappropriate nutritional status of college students. The most lacking nutrients reported in the typical college-student's diet are fiber, vitamin $\mathrm{D}$, vitamin $\mathrm{E}$, calcium, magnesium, potassium, and iron (35). Shimbo et al studied students' diet in Japan and showed calcium and iron deficiency in their diet (36). Low mineral and vitamins intake have been mentioned in Iranian student's diet. For example, Tarighat et al indicated calcium and magnesium deficiency of students of Ardabil University of Medical Sciences (37).

\section{Limitations of the Study}

Limitations of the study are the small number of participants; additional studies may be needed using a wider geographic scope and a larger sample size. The study was conducted only in dormitories in an urban setting, which may not be generalized to other girls from rural populations and lower social class since they may not have been adequately represented. In this study 2 students did not complete the questionnaires and were excluded from analyses. The need for regular and daily taking of the pills may be frustrating that causes loss to follow-up.

In this study, we did not measure calcium and magnesium intake of the participants. Significant effect of the interventions on the pain relieve observed in this study may be related to calcium and magnesium deficiency in diet of the study subjects and the results may not be generalized to women with sufficient diet. Therefore, further study with measurement of nutritional status of participants and also on women with no dietary deficiency could provide comprehensive information in this area.

Another limitation of this study is the use of self-reporting questionnaires and relatively short duration of the study and no follow-up after discontinuation of supplementation. Based on results of this study, we cannot judge the long-term effects of these supplements on primary dysmenorrhea.

\section{Conclusion}

Based on the findings of the present study, it can be concluded that the combined calcium-magnesium and calcium alone treatment during 15 days of cycle can reduce the intensity of menstrual pain, rest length and use of analgesics due to primary dysmenorrhea with no adverse event. The effect was higher with combined calcium and magnesium compared with calcium alone. In the light of the study results, it was recommended that complementary therapy should be incorporated in nursing practice especially provided as a pain relief measures to adolescent girls suffering of primary dysmenorrhea, and its related symptoms.

Supplemental therapy is one of the most commonly used branches of the complementary and alternative medicine. Also it was recommended to encourage the girls to increase calcium and magnesium in their food intake. It is necessity to adopt a healthy behavior, including an appropriate nutrition. Young girls should be informed about menstruation and need to be taught about ways to cope with dysmenorrhea. However, for general application of the results, further research is recommended.

\section{Ethical Issues}

The study was approved scientifically by the research committee of Tabriz University of Medical Sciences and ethically by the ethic committee of the university and it was registered in the Iranian Registry of Clinical Trials website before starting participant recruitment (Identifier: IRCT201202013706N10).

\section{Conflict of Interests}

Author declare that they have no competing interests.

\section{Financial Support}

None.

\section{Acknowledgments}

We would like to thank all of the students who participated in this trial.

\section{References}

1. Rapkin AJ, Nathan L. Pelvic Pain and Dysmenorrhea. In: Berek J, ed. Berek and Novak's Gynecology. Baltimore: Lippincott Williams and Wilkins; 2012:506-534.

2. French L. Dysmenorrhea. Am Fam Physician. 2005;71(2):285-291.

3. Hayes EC, Rock JA. COX-2 inhibitors and their role in gynecology. Obstet Gynecol Surv. 2002;57(11):768-780.

4. Morrison BW, Daniels SE, Kotey P, Cantu N, Seidenberg B. Rofecoxib, a specific cyclooxygenase-2 inhibitor, in primary dysmenorrhea: a randomized controlled trial. Obstet Gynecol. 1999;94(4):504-508.

5. Smith WL, Langenbach R. Why there are two cyclooxygenase isozymes. J Clin Invest 2001;107(12):1491-1495.

6. Proctor ML, Murphy PA. Herbal and dietary therapies for primary and secondary dysmenorrhoea. Cochrane Database Syst Rev. 2001;(3):CD002124.

7. Penland JG, Johnson PE. Dietary calcium and manganese effects on menstrual cycle symptoms. Am J Obstet Gynecol. 1993;168(5):1417-1423.

8. Thys-Jacobs S, Ceccarelli S, Bierman A, Weisman H, Cohen MA, Alvir J. Calcium supplementation in premenstrual syndrome: a randomized crossover trial. J Gen Intern Med. 1989;4(3):183-189.

9. Thys-Jacobs S, Starkey P, Bernstein D, Tian J. Calcium 
carbonate and the premenstrual syndrome: effects on premenstrual and menstrual symptoms. Am J Obstet Gynecol. 1998;179(2):444-452.

10. Alvir JM, Thys-Jacobs S. Premenstrual and menstrual symptom clusters and response to calcium treatment. Psychopharmacol Bull. 1991;27(2):145-148.

11. Thys-Jacobs S. Micronutrients and the premenstrual syndrome: the case for calcium. J Am Coll Nutr 2000;19(2):220-227.

12. Cunningham FG, Leveno KJ, Bloom SL, Hauth JC, Rouse DJ, Spong CY. Williams Obstetrics. New York: McGrawHill; 2010.

13. Langley WF, Mann D. Central nervous system magnesium deficiency. Arch Intern Med. 1991;151(3):593-596.

14. Benassi L, Barletta FP, Baroncini L, et al. Effectiveness of magnesium pidolate in the prophylactic treatment of primary dysmenorrhea. Clin Exp Obstet Gynecol. 1992;19(3):176-179.

15. Facchinetti F, Borella P, Sances G, Fioroni L, Nappi RE, Genazzani AR. Oral magnesium successfully relieves premenstrual mood changes. Obstet Gynecol. 1991;78(2):177-181.

16. Walker AF, De Souza MC, Vickers MF, Abeyasekera S, Collins ML, Trinca LA. Magnesium supplementation alleviates premenstrual symptoms of fluid retention. J Womens Health. 1998;7(9):1157-1165.

17. Fontana-Klaiber H, Hogg B. Therapeutic effects of magnesium in dysmenorrhea. Schweiz Rundsch Med Prax. 1990;79(16):491-4. [German].

18. Seifert B, Wagler P, Dartsch S, Schmidt U, Nieder J. Magnesium--a new therapeutic alternative in primary dysmenorrhea. Zentralbl Gynakol. 1989;111(11):755-760. [German].

19. Marjoribanks J, Proctor ML, Farquhar C. Nonsteroidal anti-inflammatory drugs for primary dysmenorrhoea. Cochrane Database Syst Rev. 2003;(4):CD001751.

20. Abraham GE. Nutritional factors in the etiology of the premenstrual tension syndromes. J Reprod Med. 1983;28(7):446-464.

21. Flink EB. Magnesium deficiency. Etiology and clinical spectrum. Acta Med Scand Suppl. 1981;647:125-137.

22. Graber TW, Yee AS, Baker FJ. Magnesium: physiology, clinical disorders, and therapy. Ann Emerg Med. 1981;10(1):49-57.

23. Davis LS. Stress, vitamin B6 and magnesium in women with and without dysmenorrhea: a comparison and intervention study [dissertation]. Austin (TX): University of Texas at Austin: 1988.

24. Franchignoni F, Salaffi F, Tesio L. How should we use the visual analogue scale (VAS) in rehabilitation outcomes? I: How much of what? The seductive VAS numbers are not true measures J Rehabil Med. 2012;44(9):798-799. doi: 10.2340/16501977-1030.

25. Drosdzol A, Skrzypulec V. Dysmenorrhea in pediatric and adolescent gynaecology. Ginekol Pol. 2008;79(7):499-503. [Polish].

26. Dawood MY. Nonsteroidal anti-inflammatory drugs and changing attitudes toward dysmenorrhea. Am J Med. 1988;84(5A):23-29.

27. Andrea J, Candace N. Pelvic Pain and Dysmenorrhea. In: Novak E, Berek JS. Berek \& Novak's Gynecology. 14th ed. Philadelphia: Lippincott Williams \& Wilkins; 2007:506534.

28. Dennehy CE. The use of herbs and dietary supplements in gynecology: an evidence-based review. J Midwifery Womens Health. 2006;51(6):402-409.

29. Muneyvirci-Delale O, Nacharaju VL, Altura BM, Alutra BT. Sex steroid hormones modulate serum ionized magnesium and calcium levels throughout the menstrual cycle in women. Fertil Steril. 1998;69(5):958-962.

30. Thys-Jacobs S, Mcmahon D, Bilezikian JP. Cyclical changes in calcium metabolism across the menstrual cycle in women with premenstrual dysphoric disorder. J Clin Endocrinol Metab. 2007;92(8):2952-2959.

31. Di Cintio E, Parazzini F, Tozzi L, et al. Dietary habits, reproductive and menstrual factors and risk of dysmenorrhoea. Eur J Epidemiol. 1997;13(8):925-930.

32. Hudson T. Using nutrition to relieve primary dysmenorrhea. Altern Complement Ther. 2007;13(3):125-128.

33. Walker AF, Marakis G, Christie S, Byng M. Mg citrate found more bioavailable than other $\mathrm{Mg}$ preparations in a randomised, double-blind study. Magnes Res. 2003;16(3):183-191.

34. Del Valle HB, Yaktine AL, Taylor CL, Ross AC, editors. Dietary reference intakes for calcium and vitamin D. National Academies Press; 2011.

35. Yu C. Contribution of Dietary Supplements to the Nutritional Status of College Students (dissertation). Connecticut: University of Connecticut; 2011

36. Shimbo S, Zhang ZW, Matsuda-Inoguchi N, et al. Effects of life away from home and physical exercise on nutrient intake and blood/serum parameters among girl students in Japan. Tohoku J Exp Med. 2004;203(4):275-286.

37. Tarighat A, Mahdavi R, Ghaemmaghami J, Saafaian A. Comparing nutritional status of college female students living on and off campus in Ardabil, 1999. J Ardabil Univ Med Sci. 2003;3(3):44-52. [Persian].

Copyright ( 2017 The Author (s); This is an open-access article distributed under the terms of the Creative Commons Attribution License (http://creativecommons.org/licenses/by/4.0), which permits unrestricted use, distribution, and reproduction in any medium, provided the original work is properly cited. 